\title{
Application of Fluorine-18-Deoxyglucose Positron Emission Tomography and Gallium Scan for Assessment in a Patient With Adult-Onset Still's Disease
}

\author{
Jing-Uei HOU, Shih-Chuan TSAI, Wan-Yu LIN \\ Department of Nuclear Medicine, Taichung Veterans General Hospital, Taichung, Taiwan
}

\begin{abstract}
A 53-year-old female patient suffered from pain in almost her entire body, particularly the joints. Chest computed tomography revealed multiple lymphadenopathies over cervical, mediastinal, and axillary areas. A fluorine-18-deoxyglucose (FDG) positron emission tomography/computed tomography (PET/CT) revealed increased FDG uptake in many lymph nodes and the spleen. Lymphoma was suspected. However, the result of a biopsy showed no malignancy, and the gallium- 67 citrate scan showed no gallium-avid tumor throughout the whole body. Adult-onset Still's disease was diagnosed and the patient responded well to steroid therapy. The follow-up PET/CT six months later showed complete remission of the FDG-avid lesions seen in the previous PET/CT. Our study suggests that FDG PET/CT combined with gallium- 67 scan may be helpful in diagnosing patients with adult-onset Still's disease. In addition, the use of FDG PET/CT alone may be useful for the evaluation of disease distribution, disease activity, and therapeutic response.

Keywords: Adult-onset Still's disease; fluorine-18-deoxyglucose; gallium-67; positron emission tomography/computed tomography.
\end{abstract}

Adult-onset Still's disease (AOSD) is an inflammatory disorder of unknown cause. "Still's disease" was first described in children by George Still in $1896 .{ }^{1}$ In 1971, the term "Adult-onset Still's disease" was used to describe patients who had variable systemic features. ${ }^{2}$ Yamaguchi et al. $^{3}$ have proposed that the classification of AOSD requires the presence of five or more criteria, of which at least two are major criteria. Major criteria: (i) Temperature of $>39{ }^{\circ} \mathrm{C}$ for $>1$ week. (ii) Leukocytosis $>10,000 / \mathrm{mm}^{3}$ with $>80 \%$ polymorphonuclear neutrophils (PMNs). (iii) Typical rash. (iv) Arthralgia $>2$ weeks. Minor criteria: (i) Sore throat. (ii) Lymph node enlargement. (iii) Splenomegaly. (iv) Liver dysfunction (high aspartate aminotransferase/ alanine aminotransferase). (v) Negative antinuclear antibodies, rheumatoid factor. ${ }^{3}$ Before establishing a final diagnosis of AOSD, other malignancies and rheumatic diseases should be ruled out. ${ }^{4}$

\section{CASE REPORT}

A 53-year-old female patient suffered from pain over most of her body, particularly the joints and the throat. In addition, skin rashes on the trunk without pruritus were noted. She had visited the Rheumatology, Neurology, and Orthopedic Outpatient Department several times. A chest computed tomography (CT) was performed which revealed multiple lymphadenopathies over cervical, mediastinal, and axillary areas. A positron emission tomography (PET)/CT revealed increased fluorine-18-deoxyglucose (FDG) uptake in many lymph nodes including the bilateral neck, axillae, mediastinum, bilateral pulmonary hili, abdominal paraaortic region, iliac regions, and inguinal regions as well as the bone marrow and the spleen (Figure 1). In addition, mild and diffuse increase of FDG uptake in the bone marrow was noted. Lymphoma was 
suspected first. After discussing with the patient, thoracoscopic mediastinal lymph node biopsy was performed. Fifteen lymph nodes were taken and the results showed histiocytic infiltration in sinusoid area and anthracotic pigment deposition. Reactive lymphoid follicle hyperplasia and focal fibrocalcified nodule formation were also noted. No tumor cell was seen. Gallium-67 (Ga-67) scan was conducted and no gallium-avid tumor was seen throughout the whole body (Figure 2). The laboratory data were negative for antinuclear antibodies, rheumatoid factor- immunoglobulin $\mathrm{M}$, and extractable nuclear antibody, while alanine aminotransferase was high. The final diagnosis was AOSD. In our case, there were no symptoms or signs of either high fever or debilitating joint although the FDG PET showed multiple FDG-avid lesions in the whole body scan. According to the experience of our rheumatologists, methylprednisolone (Metisone) $4 \mathrm{mg} /$ day and non-steroidal anti-inflammatory drugs were prescribed initially and the response to treatment was good. Six months later, the followup PET/CT showed complete remission of the FDG-avid lesions seen in the previous PET/CT (Figure 1) and the patient's symptoms relieved. A written informed consent was obtained from the patient.

\section{DISCUSSION}

Adult-onset Still's disease is a chronic systemic inflammatory disorder. Other malignancies and rheumatic diseases should be ruled out first before a diagnosis of AOSD is established. The etiology of AOSD remains unknown. Both infectious etiology and genetic factors have been suggested, although not confirmed. AOSD is characterized by spiking fever, leukocytosis, rash, and arthralgia. Some patients may also develop sore throat, lymphadenopathies, and hepatosplenomegaly. ${ }^{5-8}$ The therapeutic decision depends on the severity of disease and subsequent treatment decisions should be made in accordance with the clinical response. If the symptoms are mild, patients may respond to non-steroidal anti-inflammatory drugs. However, some still need low-dose glucocorticoid therapy to control the inflammatory response. If the symptoms are moderate such as high fever or debilitating joint, patients should receive glucocorticoid treatment initially. If the symptoms are more severe such as organ involvement which may be life-threatening, high dose glucocorticoids or even pulse therapy should be considered. ${ }^{9-12}$

In this study, we present the FDG PET/CT and Ga-67 scan findings of a patient who met the Yamaguchi classification criteria for AOSD. The FDG PET/CT scan was not helpful in diagnosing AOSD in this case since lymphoma could not be ruled out. However, the FDG PET/CT scan results were useful for evaluating the distribution
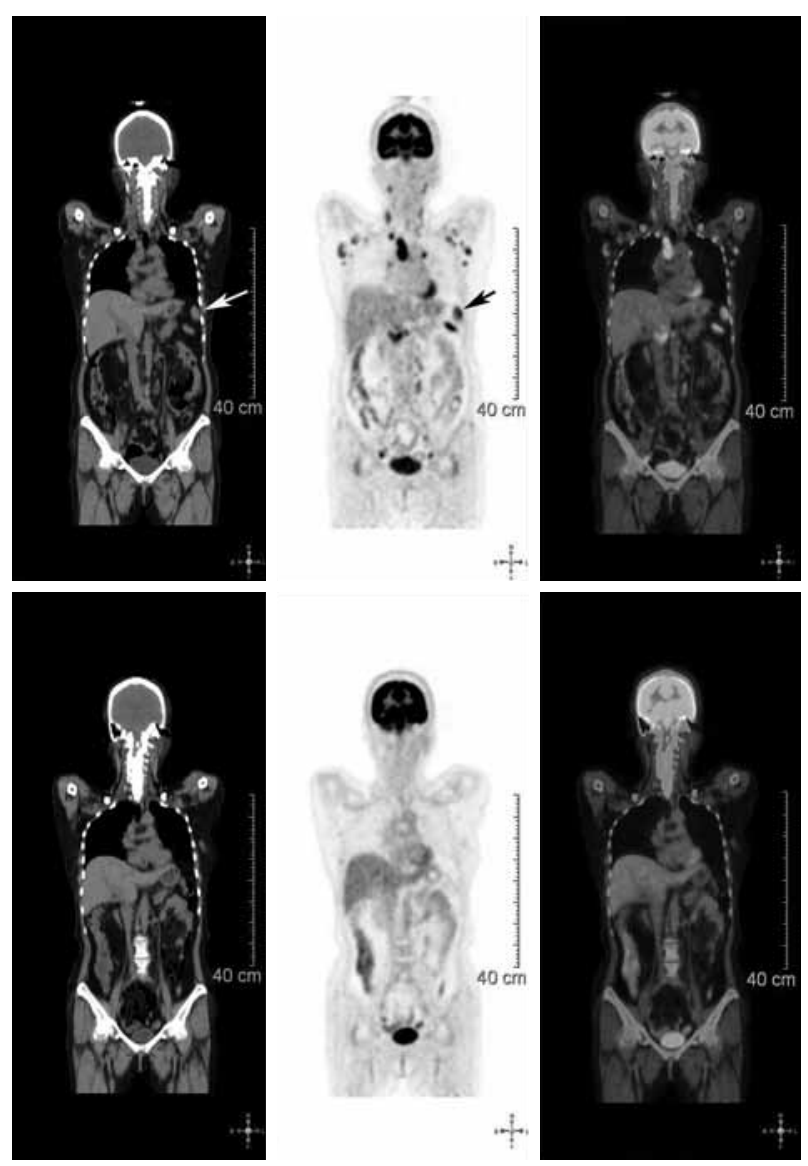

Figure 1. F18-fluorine-18-deoxyglucose positron emission tomography/computed tomography scan performed before treatment showed (upper row) increased fluorine18-deoxyglucose uptake at bilateral neck, axillae, mediastinum, bilateral pulmonary hili, abdominal para-aortic, iliac regions, and inguinal regions, and also in spleen (arrow). Lymphoma involving aforementioned areas was considered first. However, a biopsy was performed and no evidence of malignancy was found. Adult-onset Still's disease was diagnosed and patient received medical treatment. Six months later, follow-up F18-fluorine-18deoxyglucose positron emission tomography/computed tomography scan (lower row) showed complete remission of the fluorine-18-deoxyglucose-avid lesions. 


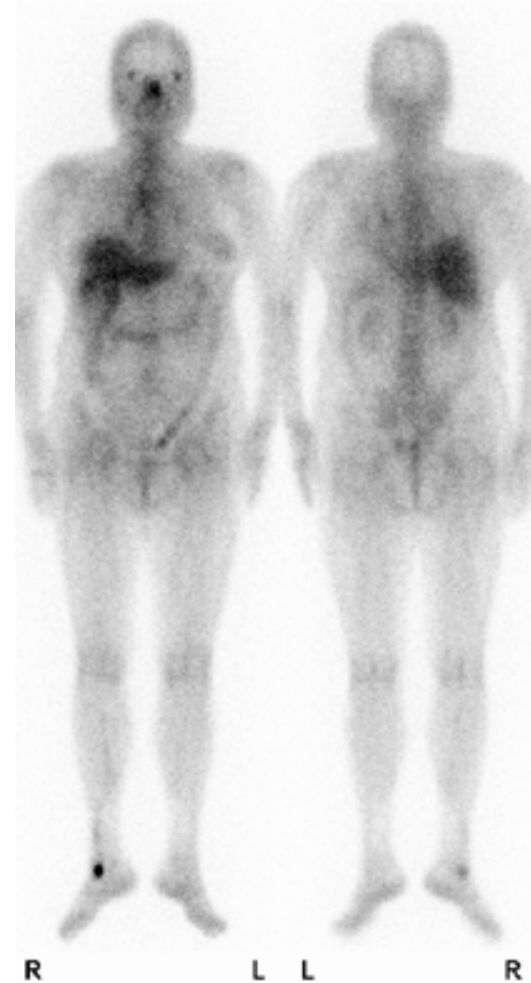

Figure 2. A gallium-67 scan performed before treatment showed no gallium-avid tumor in whole body scan.

and severity of the disease. A negative Ga-67 scan could be beneficial as it would exclude lymphoma. After the diagnosis of AOSD was established, the patient received nonsteroidal anti-inflammatory drugs and glucocorticoids with a good response. Disease activity and therapeutic response were monitored by FDG PET/CT and the findings of a scan performed six months after treatment were negative. ${ }^{13}$

In this case, we were unable to diagnose bone marrow involvement since no bone marrow biopsy was performed. There was mild and diffuse increase of FDG uptake in the bone marrow with a maximum standard uptake value of 2.5 in the spines, which was similar to the FDG uptake in the liver. According to a study by Inoue et al., ${ }^{14}$ a bone marrow F-18 FDG uptake greater than or equal to that of the liver may indicate bone marrow hyperactivity. Therefore, bone marrow involvement could not be ruled out in this case. The PET/CT six months later revealed that the maximum standard uptake value decreased to 2.0

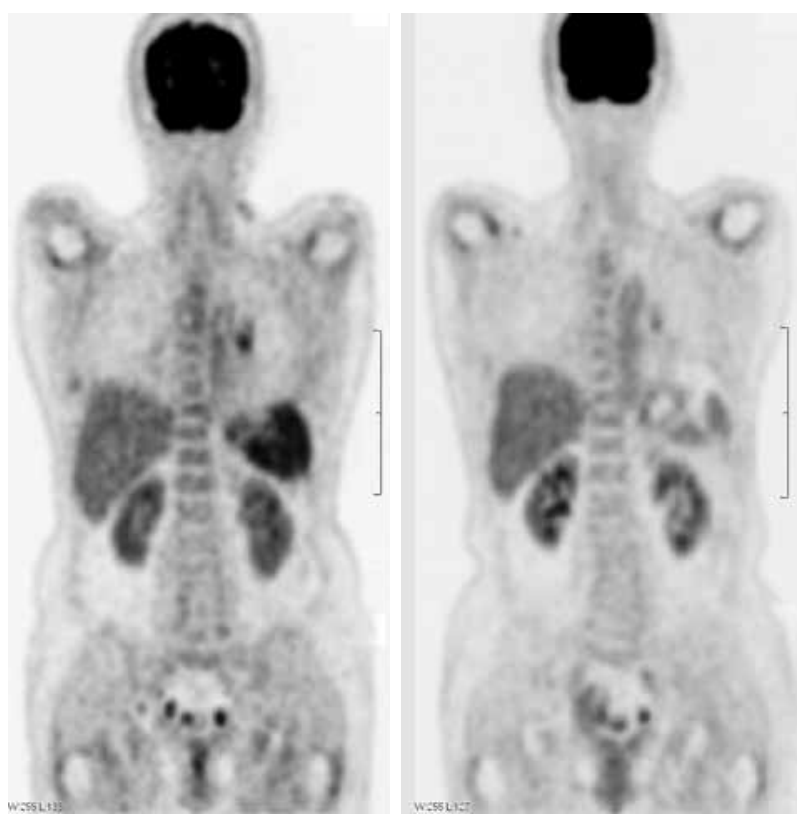

Figure 3. F18-fluorine-18-deoxyglucose positron emission tomography/computed tomography scan performed before treatment (left) showed mildly and diffusely increased fluorine-18-deoxyglucose uptake in spines (maximum standard uptake value $=2.5$ ). Six months later, follow-up F18-fluorine-18-deoxyglucose positron emission tomography/computed tomography scan (right) showed lower fluorine-18-deoxyglucose uptake in spines (maximum standard uptake value $=2.0$ ).

in the spines and was less than that in the liver (Figure 3).

The treatment of AOSD remains largely empirical, relying so far on a few prospective or retrospective studies and not on doubleblinded randomized trials with suitable sample sizes. ${ }^{12}$ In our case, there were no symptoms or signs of high fever, debilitating joint or lifethreatening complications although the FDG PET showed multiple FDG-avid lesions in the whole body scan. According to the experience of our rheumatologists, low dose of corticosteroids was prescribed first and the response to treatment was good. The patient's symptoms and signs relieved and the follow-up PET/CT showed complete remission of these FDG-avid lesions six months later.

In conclusion, there is no definitive diagnostic imaging study for patients with AOSD. Our study suggests that FDG PET/CT combined with Ga-67 scan may be helpful in diagnosing patients with AOSD. In addition, the use of FDG PET/CT 
alone may be useful for the evaluation of disease distribution, disease activity, and therapeutic response.

\section{Declaration of conflicting interests}

The authors declared no conflicts of interest with respect to the authorship and/or publication of this article.

\section{Funding}

The authors received no financial support for the research and/or authorship of this article.

\section{REFERENCES}

1. Still GF. On a Form of Chronic Joint Disease in Children. Med Chir Trans 1897;80:47-60.9.

2. Bywaters EG. Still's disease in the adult. Ann Rheum Dis 1971;30:121-33.

3. Yamaguchi M, Ohta A, Tsunematsu T, Kasukawa R, Mizushima Y, Kashiwagi H, et al. Preliminary criteria for classification of adult Still's disease. J Rheumatol 1992;19:424-30.

4. Kádár J, Petrovicz E. Adult-onset Still's disease. Best Pract Res Clin Rheumatol 2004;18:663-76.

5. Efthimiou P, Paik PK, Bielory L. Diagnosis and management of adult onset Still's disease. Ann Rheum Dis 2006;65:564-72.

6. Riera E, Olivé A, Narváez J, Holgado S, Santo P,
Mateo L, et al. Adult onset Still's disease: review of 41 cases. Clin Exp Rheumatol 2011;29:331-6.

7. Mert A, Ozaras R, Tabak F, Bilir M, Ozturk R, Ozdogan $\mathrm{H}$, et al. Fever of unknown origin: a review of 20 patients with adult-onset Still's disease. Clin Rheumatol 2003;22:89-93.

8. Elkon KB, Hughes GR, Bywaters EG, Ryan PF, Inman $\mathrm{RD}$, Bowley NB, et al. Adult-onset Still's disease. Twenty-year followup and further studies of patients with active disease. Arthritis Rheum 1982;25:647-54.

9. Kontzias A, Efthimiou P. Adult-onset Still's disease: pathogenesis, clinical manifestations and therapeutic advances. Drugs 2008;68:319-37.

10. Fautrel B. Adult-onset Still disease. Best Pract Res Clin Rheumatol 2008;22:773-92.

11. Pouchot J, Arlet JB. Biological treatment in adult-onset Still's disease. Best Pract Res Clin Rheumatol2012;26:477-87.

12. Gerfaud-Valentin M, Maucort-Boulch D, Hot A, Iwaz J, Ninet J, Durieu I, et al. Adult-onset still disease: manifestations, treatment, outcome, and prognostic factors in 57 patients. Medicine (Baltimore) 2014;93:91-9.

13. Choe JY, Chung DS, Park SH, Kwon HH, Kim SK. Clinical significance of 18F-fluoro-dexoxyglucose positron emission tomography in patients with adultonset Still's disease: report of two cases and review of literatures. Rheumatol Int 2010;30:1673-6.

14. Inoue $\mathrm{K}$, Goto R, Okada K, Kinomura S, Fukuda H. A bone marrow F-18 FDG uptake exceeding the liver uptake may indicate bone marrow hyperactivity. Ann Nucl Med 2009;23:643-9. 